\title{
A new and stronger central sets theorem
}

\author{
by \\ Dibyendu De (Krishnagar), \\ Neil Hindman (Washington, DC) and Dona Strauss (Leeds)
}

\begin{abstract}
Furstenberg's original Central Sets Theorem applied to central subsets of $\mathbb{N}$ and finitely many specified sequences in $\mathbb{Z}$. In this form it was already strong enough to derive some very strong combinatorial consequences, such as the fact that a central subset of $\mathbb{N}$ contains solutions to all partition regular systems of homogeneous equations. Subsequently the Central Sets Theorem was extended to apply to arbitrary semigroups and countably many specified sequences. In this paper we derive a new version of the Central Sets Theorem for arbitrary semigroups $S$ which applies to all sequences in $S$ at once. We show that the new version is strictly stronger than the original version applied to the semigroup $(\mathbb{R},+)$. And we show that the noncommutative versions are strictly increasing in strength.
\end{abstract}

1. Introduction. In [3] Furstenberg defined a central subset of the set $\mathbb{N}$ of positive integers in terms of some notions from topological dynamics. He showed that if $\mathbb{N}$ is partitioned into finitely many classes, one of these classes contains a central set. Then he proved the following theorem. (For any set $X$, we write $\mathcal{P}_{\mathrm{f}}(X)$ for the set of finite nonempty subsets of $X$.)

1.1. The Original Central Sets Theorem (Furstenberg). Let $l \in \mathbb{N}$ and for each $i \in\{1, \ldots, l\}$, let $\left\langle y_{i, n}\right\rangle_{n=1}^{\infty}$ be a sequence in $\mathbb{Z}$. Let $C$ be a central subset of $\mathbb{N}$. Then there exist sequences $\left\langle a_{n}\right\rangle_{n=1}^{\infty}$ in $\mathbb{N}$ and $\left\langle H_{n}\right\rangle_{n=1}^{\infty}$ in $\mathcal{P}_{\mathrm{f}}(\mathbb{N})$ such that

(1) for all $n, \max H_{n}<\min H_{n+1}$,

(2) for all $F \in \mathcal{P}_{\mathrm{f}}(\mathbb{N})$ and all $i \in\{1, \ldots, l\}, \sum_{n \in F}\left(a_{n}+\sum_{t \in H_{n}} y_{i, t}\right) \in C$.

Proof. [3, Proposition 8.21].

He pointed out that an immediate consequence is that whenever $\mathbb{N}$ is divided into finitely many classes, and a sequence $\left\langle x_{n}\right\rangle_{n=1}^{\infty}$ is given, one of

2000 Mathematics Subject Classification: Primary 05D10; Secondary 54H13, 22A15.

Key words and phrases: central set of integers.

N. Hindman acknowledges support received from the National Science Foundation via Grant DMS-0554803. 
the classes must contain arbitrarily long arithmetic progressions with the increment $d \in \operatorname{FS}\left(\left\langle x_{n}\right\rangle_{n=1}^{\infty}\right)=\left\{\sum_{n \in F} x_{n}: F \in \mathcal{P}_{\mathrm{f}}(\mathbb{N})\right\}$. (To see this, let $l \in \mathbb{N}$ and for $i \in\{0,1, \ldots, l\}$ let $y_{i, n}=i \cdot x_{n}$. Pick a cell of the partition which contains a central set $C$ and pick $\left\langle a_{n}\right\rangle_{n=1}^{\infty}$ and $\left\langle H_{n}\right\rangle_{n=1}^{\infty}$ as guaranteed by the Central Sets Theorem. Now throw away all but the first term of each sequence. Let $d=\sum_{t \in H_{1}} x_{t}$. Then for $i \in\{0,1, \ldots, l\}, a_{1}+i d=$ $a_{1}+\sum_{t \in H_{1}} y_{i, t} \in C$.) Furstenberg also used central sets to prove Rado's theorem [7] by showing that any central subset of $\mathbb{N}$ contains solutions to all partition regular systems of homogeneous linear equations.

Subsequently, after looking at an early draft of the paper [4] by Furstenberg and Katznelson which derived Ramsey-theoretic results using idempotents in enveloping semigroups, Vitaly Bergelson had the idea that one might be able to derive the conclusion of the Central Sets Theorem for a set $C \subseteq \mathbb{N}$ which had an idempotent in the smallest ideal of $\beta \mathbb{N}$ in its closure. (Here $\beta \mathbb{N}$ is the Stone-Cech compactification of $\mathbb{N}$. We shall present a brief introduction to its structure later in this section.) He was right. This suggested the following definition which makes sense in any semigroup.

1.2. Definition. Let $S$ be a discrete semigroup and let $C$ be a subset of $S$. Then $C$ is central if and only if there is an idempotent $p$ in the smallest ideal of $\beta S$ such that $p \in \operatorname{cl} C$.

In [1] it was shown, with the assistance of B. Weiss, that a subset $C$ of $\mathbb{N}$ is central according to Definition 1.2 if and only if $C$ is central according to Furstenberg's original definition. Furstenberg's original definition extends naturally to an arbitrary semigroup and in [8] Hong-ting Shi and Hong-wei Yang showed that this extended definition is equivalent to that of Definition 1.2.

In [2], the Central Sets Theorem was extended to arbitrary semigroups. The version for commutative semigroups extended Theorem 1.1 by allowing the choice of the sequence which was used to vary as $n$ varied. (We shall deal with noncommutative versions later.) For purposes of comparison with the noncommutative versions we introduce the following notation.

1.3. Definition. Let $(S,+)$ be a commutative semigroup, let $a \in S$, let $H \in \mathcal{P}_{\mathrm{f}}(\mathbb{N})$, and let $\left\langle y_{t}\right\rangle_{t=1}^{\infty}$ be a sequence in $S$. Then $x\left(a, H,\left\langle y_{i}\right\rangle_{t=1}^{\infty}\right)=$ $a+\sum_{t \in H} y_{t}$.

With this notation, conclusion (2) of Theorem 1.1 becomes "for all $F \in$ $\mathcal{P}_{\mathrm{f}}(\mathbb{N})$ and all $i \in\{1, \ldots, l\}, \sum_{n \in F} x\left(a_{n}, H_{n},\left\langle y_{i, t}\right\rangle_{t=1}^{\infty}\right) \in C . "$

1.4. Theorem. Let $(S,+)$ be a commutative semigroup. Let $l \in \mathbb{N}$ and for each $i \in\{1, \ldots, l\}$, let $\left\langle y_{i, n}\right\rangle_{n=1}^{\infty}$ be a sequence in $S$. Let $C$ be a central subset of $S$. Then there exist sequences $\left\langle a_{n}\right\rangle_{n=1}^{\infty}$ in $S$ and $\left\langle H_{n}\right\rangle_{n=1}^{\infty}$ in $\mathcal{P}_{\mathrm{f}}(\mathbb{N})$ such that 
(1) for all $n, \max H_{n}<\min H_{n+1}$,

(2) for all $F \in \mathcal{P}_{\mathrm{f}}(\mathbb{N})$ and $f: F \rightarrow\{1, \ldots, l\}, \sum_{n \in F} x\left(a_{n}, H_{n},\left\langle y_{f(n), t}\right\rangle_{t=1}^{\infty}\right)$ $\in C$.

Proof. [2, Corollary 2.10].

The alert reader may have noticed that in Theorem 1.1, $C$ is central in $\mathbb{N}$ while the sequences $\left\langle y_{i, n}\right\rangle_{n=1}^{\infty}$ are allowed to come from $\mathbb{Z}$. It is a fact, which follows from [6, Exercise 4.3.5 and Theorem 1.65], that any set central in $(\mathbb{N},+)$ is also central in $(\mathbb{Z},+)$, so Theorem 1.1 does follow from Theorem 1.4.

In [6] we extended the Central Sets Theorem further by dealing with countably many sequences at a time. The straightforward extension of Theorem 1.4 to countably many sequences (in which conclusion (2) would read "for all $F \in \mathcal{P}_{\mathrm{f}}(\mathbb{N})$ and all $f: F \rightarrow \mathbb{N}, \sum_{n \in F} x\left(a_{n}, H_{n},\left\langle y_{f(n), t}\right\rangle_{t=1}^{\infty}\right) \in C$ ") is not valid. One can see this because it would easily imply that any central set in $\mathbb{N}$, and thus one cell of any finite partition of $\mathbb{N}$, would contain infinite arithmetic progressions. One needs to restrict oneself to dealing with finitely many sequences at one time, so we use the following set of functions. Given sets $X$ and $Y$, we write ${ }^{X} Y$ for the set of functions from $X$ to $Y$.

1.5. Definition. $\Phi=\left\{f \in \mathbb{N}_{\mathbb{N}}\right.$ : for all $\left.n \in \mathbb{N}, f(n) \leq n\right\}$.

1.6. Theorem. Let $(S,+)$ be a commutative semigroup and for each $i \in \mathbb{N}$, let $\left\langle y_{i, n}\right\rangle_{n=1}^{\infty}$ be a sequence in $S$. Let $C$ be a central subset of $S$. Then there exist sequences $\left\langle a_{n}\right\rangle_{n=1}^{\infty}$ in $S$ and $\left\langle H_{n}\right\rangle_{n=1}^{\infty}$ in $\mathcal{P}_{\mathrm{f}}(\mathbb{N})$ such that

(1) for all $n, \max H_{n}<\min H_{n+1}$,

(2) for all $F \in \mathcal{P}_{\mathrm{f}}(\mathbb{N})$ and $f \in \Phi, \sum_{n \in F} x\left(a_{n}, H_{n},\left\langle y_{f(n), t}\right\rangle_{t=1}^{\infty}\right) \in C$.

Proof. [6, Theorem 14.11].

In this paper we prove an extension of the Central Sets Theorem for commutative semigroups which applies to all sequences in $S$ at once and we prove the corresponding extension for the Central Sets Theorem for noncommutative semigroups.

In Section 2 we shall derive the new commutative version. We shall also show that there exist commutative semigroups, including $(\mathbb{R},+)$, in which the conclusion of Theorem 1.4 is strictly stronger than the obvious generalization of Theorem 1.1 to arbitrary commutative semigroups.

In Section 3 we shall derive the new noncommutative version and investigate those members of $\beta S$ all of whose members satisfy the new Central Sets Theorem.

In Section 4 we shall show that in the free semigroup on $\omega_{1}$ generators the new Central Sets Theorem is strictly stronger than the noncommutative version of Theorem 1.6. We shall also show in that section that in the free semigroup on $\mathfrak{c}$ generators, the noncommutative version of Theorem 1.6 is 
strictly stronger than the noncommutative version of Theorem 1.4, which is in turn strictly stronger than the noncommutative version of Theorem 1.1.

We now present a very brief review of basic facts about $(\beta S, \cdot)$. For additional information see [6].

Given a discrete semigroup $(S, \cdot)$ we take the points of the Stone-Cech compactification $\beta S$ of $S$ to be the ultrafilters on $S$, the principal ultrafilters being identified with the points of $S$. Given $A \subseteq S, \bar{A}=\{p \in \beta S: A \in p\}$, and the set $\{\bar{A}: A \subseteq S\}$ is a basis for the open sets (and a basis for the closed sets) of $\beta S$. Given $p, q \in \beta S$ and $A \subseteq S, A \in p \cdot q$ if and only if $\left\{x \in S: x^{-1} A \in q\right\} \in p$, where $x^{-1} A=\{y \in S: x \cdot y \in A\}$. In particular, the operation * on $\beta S$ extends the operation - on $S$. If the operation is denoted by + , then $A \in p+q$ if and only if $\{x \in S:-x+A \in q\} \in p$. The reader should be warned however, that even if $S$ is commutative, $\beta S$ seldom is. In particular, the algebraic centers of $(\beta \mathbb{N}, \cdot)$ and $(\beta \mathbb{N},+)$ are both equal to $\mathbb{N}$.

With this operation, $(\beta S, \cdot)$ is a compact Hausdorff right topological semigroup with $S$ contained in its topological center. That is, for each $p$ in $\beta S$, the function $\varrho_{p}: \beta S \rightarrow \beta S$ defined by $\varrho_{p}(q)=q \cdot p$ is continuous and for each $x \in S$, the function $\lambda_{x}: \beta S \rightarrow \beta S$ defined by $\lambda_{x}(q)=x \cdot q$ is continuous. A nonempty subset $I$ of a semigroup $T$ is a left ideal provided $T \cdot I \subseteq I$, a right ideal provided $I \cdot T \subseteq I$, and a two-sided ideal (or simply an $i d e a l)$ provided it is both a left ideal and a right ideal.

Any compact Hausdorff right topological semigroup $T$ has a smallest two-sided ideal $K(T)=\bigcup\{L: L$ is a minimal left ideal of $T\}=\bigcup\{R: R$ is a minimal right ideal of $T\}$. Given a minimal left ideal $L$ and a minimal right ideal $R, L \cap R$ is a group, and in particular contains an idempotent. An idempotent in $K(T)$ is a minimal idempotent. If $p$ and $q$ are idempotents in $T$ we write $p \leq q$ if and only if $p q=q p=p$. An idempotent is minimal with respect to this relation if and only if it is a member of the smallest ideal.

Thus a subset $C$ of $S$ is central if and only if it is a member of a minimal idempotent of $\beta S$.

2. The new commutative Central Sets Theorem. As with the older versions, the new Central Sets Theorem for commutative semigroups is a consequence of the general result for all semigroups. However, the commutative version is much simpler to state, and so we present its derivation separately.

We present a nearly self-contained proof, relying only on a few basic facts about compact right topological semigroups. We do this to make clear the simplicity of the proof of the new Central Sets Theorem. We begin with the following special case of Theorem 1.4. A subset $C$ of $S$ is piecewise syndetic if and only if $\bar{C} \cap K(\beta S) \neq \emptyset$. In particular, any central set is piecewise syndetic. 
As the referee pointed out, the following theorem is an immediate consequence of the corresponding result which does not require that min $H>m$. (One may simply delete the first $m$ terms of each sequence.) However, we need this version, and it is no harder to prove than the superficially more restricted version.

2.1. Theorem. Let $(S,+)$ be a commutative semigroup and let $l \in \mathbb{N}$. For each $i \in\{1, \ldots, l\}$, let $\left\langle y_{i, n}\right\rangle_{n=1}^{\infty}$ be a sequence in $S$. Let $C$ be a piecewise syndetic subset of $S$ and let $m \in \mathbb{N}$. There exist $a \in S$ and $H \in \mathcal{P}_{\mathrm{f}}(\mathbb{N})$ such that $\min H>m$ and for each $i \in\{1, \ldots, l\}, x\left(a, H,\left\langle y_{i, t}\right\rangle_{t=1}^{\infty}\right) \in C$.

Proof. Let $Y=X_{t=1}^{l} \beta S$. Then by [6, Theorem 2.22], $Y$ is a compact right topological semigroup and if $s \in X_{t=1}^{l} S$, then $\lambda_{s}$ is continuous. For $i \in \mathbb{N}$, let

$$
\begin{aligned}
& I_{i}=\left\{\left(x\left(a, H,\left\langle y_{1, t}\right\rangle_{t=1}^{\infty}\right), \ldots, x\left(a, H,\left\langle y_{l, t}\right\rangle_{t=1}^{\infty}\right)\right)\right. \\
& \left.\quad a \in S, H \in \mathcal{P}_{\mathrm{f}}(\mathbb{N}), \text { and } \min H>i\right\}
\end{aligned}
$$

and let $E_{i}=I_{i} \cup\{(a, \ldots, a): a \in S\}$.

Let $E=\bigcap_{i=1}^{\infty} \bar{E}_{i}$ and $I=\bigcap_{i=1}^{\infty} \bar{I}_{i}$. We claim that $E$ is a subsemigroup of $Y$ and $I$ is an ideal of $E$. To see this, let $p, q \in E$. We show that $p+q \in E$ and if either $p \in I$ or $q \in I$, then $p+q \in I$. Let $U$ be an open neighborhood of $p+q$ and let $i \in \mathbb{N}$. Since $\varrho_{q}$ is continuous, pick a neighborhood $V$ of $p$ such that $V+q \subseteq U$. Pick $x \in E_{i} \cap V$ with $x \in I_{i}$ if $p \in I$. If $x \in I_{i}$ so that $x=\left(x\left(a, H,\left\langle y_{1, t}\right\rangle_{t=1}^{\infty}\right), \ldots, x\left(a, H,\left\langle y_{l, t}\right\rangle_{t=1}^{\infty}\right)\right)$ for some $a \in S$ and some $H \in \mathcal{P}_{\mathrm{f}}(\mathbb{N})$ with $\min H>i$, let $j=\max H$. Otherwise, let $j=i$. Since $\lambda_{x}$ is continuous, pick a neighborhood $W$ of $q$ such that $x+W \subseteq U$. Pick $y \in E_{j} \cap W$ with $y \in I_{j}$ if $q \in I$. Then $x+y \in E_{i} \cap U$ and if either $p \in I$ or $q \in I$, then $x+y \in I_{i} \cap U$.

By [6, Theorem 2.23], $K(Y)=\chi_{t=1}^{l} K(\beta S)$. Pick $p \in K(\beta S) \cap \bar{C}$. Then $\bar{p}=(p, \ldots, p) \in K(Y)$. We claim that $\bar{p} \in E$. To see this, let $U$ be a neighborhood of $\bar{p}$, let $i \in \mathbb{N}$, and pick $A_{1}, \ldots, A_{l} \in p$ with $\chi_{t=1}^{l} \bar{A}_{t} \subseteq U$. Pick $a \in \bigcap_{t=1}^{l} A_{t}$. Then $\bar{a}=(a, \ldots, a) \in U \cap E_{i}$. Thus $\bar{p} \in K(Y) \cap E$ and consequently $K(Y) \cap E \neq \emptyset$. Then by [6, Theorem 1.65], $K(E)=$ $K(Y) \cap E$ and so $\bar{p} \in K(E) \subseteq I$. Then $I_{m} \cap \chi_{t=1}^{l} C \neq \emptyset$ so pick $z$ in $I_{m} \cap X_{t=1}^{l} C$ and pick $a \in S$ and $H \in \mathcal{P}_{\mathrm{f}}(\mathbb{N})$ with min $H>m$ such that $z=\left(x\left(a, H,\left\langle y_{1, t}\right\rangle_{t=1}^{\infty}\right), \ldots, x\left(a, H,\left\langle y_{l, t}\right\rangle_{t=1}^{\infty}\right)\right)$.

The following is the new Central Sets Theorem for commutative semigroups.

2.2. TheOREM. Let $(S,+)$ be a commutative semigroup and let $\mathcal{T}=\mathbb{N}_{S}$, the set of sequences in $S$. Let $C$ be a central subset of $S$. There exist functions $\alpha: \mathcal{P}_{\mathrm{f}}(\mathcal{T}) \rightarrow S$ and $H: \mathcal{P}_{\mathrm{f}}(\mathcal{T}) \rightarrow \mathcal{P}_{\mathrm{f}}(\mathbb{N})$ such that 
(1) if $F, G \in \mathcal{P}_{\mathrm{f}}(\mathcal{T})$ and $F \subsetneq G$, then $\max H(F)<\min H(G)$,

(2) whenever $m \in \mathbb{N}, G_{1}, \ldots, G_{m} \in \mathcal{P}_{\mathrm{f}}(\mathcal{T}), G_{1} \subsetneq \cdots \subsetneq G_{m}$, and for each $i \in\{1, \ldots, m\},\left\langle y_{i, n}\right\rangle_{n=1}^{\infty} \in G_{i}$, one has

$$
\sum_{i=1}^{m} x\left(\alpha\left(G_{i}\right), H\left(G_{i}\right),\left\langle y_{i, t}\right\rangle_{t=1}^{\infty}\right) \in C .
$$

Proof. Pick a minimal idempotent $p$ of $\beta S$ such that $C \in p$. Let

$$
C^{\star}=\{x \in C:-x+C \in p\} .
$$

As $p+p=p, C^{\star} \in p$. Also by [6, Lemma 4.14], if $x \in C^{\star}$, then $-x+C^{\star} \in p$.

We define $\alpha(F) \in S$ and $H(F) \in \mathcal{P}_{\mathrm{f}}(\mathbb{N})$ for $F \in \mathcal{P}_{\mathrm{f}}(\mathcal{T})$ by induction on $|F|$ satisfying the following inductive hypotheses:

(1) if $\emptyset \neq G \subsetneq F$, then $\max H(G)<\min H(F)$,

(2) if $n \in \mathbb{N}, \emptyset \neq G_{1} \subsetneq \cdots \subsetneq G_{n}=F$, and $\left\langle f_{i}\right\rangle_{i=1}^{n} \in X_{i=1}^{n} G_{i}$, then $\sum_{i=1}^{n} x\left(\alpha\left(G_{i}\right), H\left(G_{i}\right), f_{i}\right) \in C^{\star}$.

Assume first that $F=\{f\}$. By Theorem 2.1 pick $a \in S$ and $L \in \mathcal{P}_{\mathrm{f}}(\mathbb{N})$ such that $x\left(a, L,\langle f(t)\rangle_{t=1}^{\infty}\right) \in C^{\star}$. Let $\alpha(\{f\})=a$ and $H(\{f\})=L$.

Now assume that $|F|>1$ and $\alpha(G)$ and $H(G)$ have been defined for all proper subsets $G$ of $F$. Let $K=\bigcup\{H(G): \emptyset \neq G \subsetneq F\}$ and $m=\max K$. Let

$$
\begin{aligned}
M=\left\{\sum_{i=1}^{n} x\left(\alpha\left(G_{i}\right), H\left(G_{i}\right), f_{i}\right): n \in \mathbb{N}, \emptyset \neq G_{1} \subsetneq \cdots \subsetneq G_{n} \subsetneq F\right. & \\
& \text { and } \left.\left\langle f_{i}\right\rangle_{i=1}^{n} \in \underset{i=1}{X} G_{i}\right\} .
\end{aligned}
$$

Then $M$ is finite and by hypothesis $(2), M \subseteq C^{\star}$. Let

$$
B=C^{\star} \cap \bigcap_{x \in M}\left(-x+C^{\star}\right) .
$$

Then $B \in p$, so by Theorem 2.1 pick $a \in S$ and $L \in \mathcal{P}_{\mathrm{f}}(\mathbb{N})$ such that $\min L>m$ and for each $f \in F, x\left(a, L,\langle f(t)\rangle_{t=1}^{\infty}\right) \in B$. Let $\alpha(F)=a$ and $H(F)=L$.

Since $\min L \geq m$, hypothesis (1) is satisfied. To verify hypothesis (2), let $n \in \mathbb{N}, \emptyset \neq G_{1} \subsetneq \cdots \subsetneq G_{n}=F$, and $\left\langle f_{i}\right\rangle_{i=1}^{n} \in X_{i=1}^{n} G_{i}$. If $n=1$, then $\sum_{i=1}^{n} x\left(\alpha\left(G_{i}\right), H\left(G_{i}\right), f_{1}\right)=x\left(a, L, f_{1}\right) \in B \subseteq C^{\star}$. So assume that $n>1$ and let $y=\sum_{i=1}^{n-1} x\left(\alpha\left(G_{i}\right), H\left(G_{i}\right), f_{i}\right)$. Then $y \in M$ so $x\left(a, L, f_{n}\right) \in B \subseteq$ $\left(-y+C^{\star}\right)$ and thus $\sum_{i=1}^{n} x\left(\alpha\left(G_{i}\right), H\left(G_{i}\right), f_{i}\right)=y+x\left(a, L, f_{n}\right) \in C^{\star}$ as required.

As a simple application, we present the following corollary which is not directly derivable by a single application of Theorem 1.6. The point of the corollary is that an arithmetic progression $A$ is chosen which "works" for every length $k$ and every sequence $\left\langle y_{n}\right\rangle_{n=1}^{\infty}$. 
2.3. Corollary. Let $C$ be a central subset of $\mathbb{N}$, let $\left\langle x_{n}\right\rangle_{n=1}^{\infty}$ be a sequence in $\mathbb{N}$, and let $l \in \mathbb{N}$. There exist $a \in \mathbb{N}$ and $d \in \operatorname{FS}\left(\left\langle x_{n}\right\rangle_{n=1}^{\infty}\right)$ such that $A=\{a+d, a+2 d, \ldots, a+l d\} \subseteq C$ and whenever $\left\langle y_{n}\right\rangle_{n=1}^{\infty}$ is a sequence in $\mathbb{N}$ and $k \in \mathbb{N}$ there will exist $b \in \mathbb{N}$ and $c \in \mathrm{FS}\left(\left\langle y_{n}\right\rangle_{n=1}^{\infty}\right)$ such that $B=\{b+c, b+2 c, \ldots, b+k c\} \subseteq C$ and $A+B \subseteq C$.

Proof. Pick functions $\alpha$ and $H$ as guaranteed by the new Central Sets Theorem. Let $F=\left\{\left\langle x_{n}\right\rangle_{n=1}^{\infty},\left\langle 2 x_{n}\right\rangle_{n=1}^{\infty}, \ldots,\left\langle l x_{n}\right\rangle_{n=1}^{\infty}\right\}$. Let $a=\alpha(F)$ and let $d=\sum_{t \in H(F)} x_{t}$. Given $\left\langle y_{n}\right\rangle_{n=1}^{\infty}$ and $k$, let $G=F \cup\left\{\left\langle y_{n}\right\rangle_{n=1}^{\infty},\left\langle 2 y_{n}\right\rangle_{n=1}^{\infty}, \ldots\right.$, $\left.\left\langle k y_{n}\right\rangle_{n=1}^{\infty}\right\}$. Let $b=\alpha(G)$ and let $c=\sum_{t \in H(G)} y_{t}$.

Honesty compels us to admit that we could have derived Corollary 2.3 by two applications of Theorem 1.6, or even of Theorem 1.1, by first producing $a \in \mathbb{N}$ and $d \in \operatorname{FS}\left(\left\langle x_{n}\right\rangle_{n=1}^{\infty}\right)$ such that $A=\{a+d, a+2 d, \ldots, a+l d\} \subseteq C^{\star}$ and then applying Theorem 1.1 to the central set $\bigcap_{t=1}^{l}\left(-(a+d)+C^{\star}\right)$.

Notice that Theorem 1.6 is an easy consequence of Theorem 2.2. To see this, notice that one can assume that the sequences in the statement of Theorem 1.6 are distinct. Then given such sequences, for each $n \in \mathbb{N}$, let $F_{n}=\left\{\left\langle y_{1, t}\right\rangle_{t=1}^{\infty},\left\langle y_{2, t}\right\rangle_{t=1}^{\infty}, \ldots,\left\langle y_{n, t}\right\rangle_{t=1}^{\infty}\right\}$ and let $a_{n}=\alpha\left(F_{n}\right)$ and $H_{n}=$ $H\left(F_{n}\right)$.

We cannot prove that Theorem 2.2 is strictly stronger than Theorem 1.6 or even Theorem 1.4. (In Section 4 we will show that the corresponding noncommutative versions are indeed strictly increasing in strength.) We can, however, show that Theorem 1.4 is strictly stronger than the obvious generalization of Theorem 1.1 to arbitrary commutative semigroups which we state now.

2.4. Theorem. Let $(S,+)$ be a commutative semigroup. Let $l \in \mathbb{N}$ and for each $i \in\{1, \ldots, l\}$, let $\left\langle y_{i, n}\right\rangle_{n=1}^{\infty}$ be a sequence in $S$. Let $C$ be a central subset of $S$. Then there exist sequences $\left\langle a_{n}\right\rangle_{n=1}^{\infty}$ in $S$ and $\left\langle H_{n}\right\rangle_{n=1}^{\infty}$ in $\mathcal{P}_{\mathrm{f}}(\mathbb{N})$ such that

(1) for all $n, \max H_{n}<\min H_{n+1}$,

(2) for all $F \in \mathcal{P}_{\mathrm{f}}(\mathbb{N})$ and $i \in\{1, \ldots, l\}, \sum_{n \in F} x\left(a_{n}, H_{n},\left\langle y_{i, t}\right\rangle_{t=1}^{\infty}\right) \in C$.

The following semigroup contains much of the known algebraic structure of $\beta \mathbb{N}$ and occurs as a subsemigroup of $\beta S$ for many semigroups $S$. (See [6, especially Section 6.1].)

2.5. Definition. $\mathbb{H}=\bigcap_{n=1}^{\infty} \operatorname{cl}_{\beta \mathbb{N}}\left(2^{n} \mathbb{N}\right)$.

We shall need the following technical lemma. Recall that $\omega=\mathbb{N} \cup\{0\}$ is the first infinite ordinal.

2.6. Lemma. Let $(S,+)$ be a commutative semigroup with identity 0 and no other idempotents. Let $m \in \mathbb{N}$ and for each $i \in\{1, \ldots, m\}$, let $\left\langle y_{i, n}\right\rangle_{n=1}^{\infty}$ be a sequence in $S$. Assume that $\psi: \omega \underset{\text { onto }}{\stackrel{1-1}{\longrightarrow}} S, \psi(0)=0$, and the restriction of 
$\widetilde{\psi}$ to $\mathbb{H}$ is an injective homomorphism, where $\widetilde{\psi}: \beta \omega \rightarrow \beta S$ is the continuous extension of $\psi$. Assume further that all idempotents of $\beta S \backslash S$ are in $\widetilde{\psi}[\mathbb{H}]$. Then for each $r \in \mathbb{N}$, there exists $H \in \mathcal{P}_{\mathrm{f}}(\mathbb{N})$ such that $\min H>r$ and for all $i \in\{1, \ldots, m\}, \psi^{-1}\left(\sum_{t \in H} y_{i, t}\right) \in 2^{r} \cdot \omega$.

Proof. Consider the semigroup $\left(\mathcal{P}_{\mathrm{f}}(\mathbb{N}), \cup\right)$ and denote the extended operation in $\beta \mathcal{P}_{\mathrm{f}}(\mathbb{N})$ by $*$. (We cannot follow our usual custom of denoting the extended operation by the same symbol as used for the original semigroup since $p \cup q$ already means something.) For each $n \in \mathbb{N}$ let $B_{n}=\left\{H \in \mathcal{P}_{\mathrm{f}}(\mathbb{N})\right.$ : $\min H>n\}$. Then by [6, Theorem 4.20], $\mathcal{B}=\bigcap_{n=1}^{\infty} \operatorname{cl}_{\beta \mathcal{P}_{\mathrm{f}}(\mathbb{N})} B_{n}$ is a subsemigroup of $\left(\beta \mathcal{P}_{\mathrm{f}}(\mathbb{N}), \uplus\right)$ so pick an idempotent $p \in \mathcal{B}$. For each $i \in\{1, \ldots, m\}$ define $\theta_{i}: \mathcal{P}_{\mathrm{f}}(\mathbb{N}) \rightarrow S$ by $\theta_{i}(H)=\sum_{t \in H} y_{i, t}$.

Now let $i \in\{1, \ldots, m\}$ be given. By [6, Theorem 4.21], if $\widetilde{\theta}_{i}: \beta \mathcal{P}_{\mathrm{f}}(\mathbb{N}) \rightarrow$ $\beta S$ is the continuous extension of $\theta_{i}$, then the restriction of $\widetilde{\theta}_{i}$ to $\mathcal{B}$ is a homomorphism. Consequently, $\widetilde{\theta}_{i}(p)$ is either 0 or an idempotent in $\widetilde{\psi}[\mathbb{H}]$. Thus $\widetilde{\psi}^{-1}\left(\widetilde{\theta}_{i}(p)\right)$ is either 0 or an idempotent in $\mathbb{H}$. In any event, $\overline{2^{r} \cdot \omega}$ is a neighborhood of $\widetilde{\psi}^{-1}\left(\widetilde{\theta}_{i}(p)\right)$ in $\beta \omega$, so pick $A_{i} \in p$ such that $\widetilde{\psi}^{-1}\left(\widetilde{\theta}_{i}\left[\bar{A}_{i}\right]\right) \subseteq$ $\overline{2^{r} \cdot \omega}$.

Pick $H \in B_{r} \cap \bigcap_{i=1}^{m} A_{i}$. Then $\min H>r$ and for each $i \in\{1, \ldots, m\}$,

$$
\psi^{-1}\left(\sum_{t \in H} y_{i, t}\right)=\psi^{-1}\left(\theta_{i}(H)\right) \in 2^{r} \cdot \omega .
$$

As noted before the proof of [6, Theorem 7.28] the word "metrizable" is not really needed in the following theorem.

2.7. Theorem. For each $\iota<\mathfrak{c}$ let $\left(S_{\iota},+\right)$ be a semigroup containing $(\omega,+)$ with $\left|S_{\iota}\right| \leq \mathfrak{c}$. Assume further that either $S_{0}=\omega$ or $S_{0}$ is a countably infinite group which can be mapped into a compact metrizable group by an injective homomorphism. Let $S=\bigoplus_{\iota<\mathfrak{c}} S_{\iota}$. Then there is a subset $A$ of $S$ which satisfies the conclusion of Theorem 2.4 but not the conclusion of Theorem 1.4.

Proof. Given $\sigma<\mathfrak{c}$ define $e(\sigma) \in S$ by $e(\sigma)(\sigma)=1$ and $e(\sigma)(\iota)=0$ if $\iota \neq \sigma$. We shall use two notions of "support" in this proof. For $x \in S$, $\operatorname{supp}(x)=\{\sigma<\mathfrak{c}: x(\sigma) \neq 0\}$. For $x \in \mathbb{N}$, $\operatorname{supp}_{2}(x) \in \mathcal{P}_{\mathrm{f}}(\omega)$ is defined by $x=\sum_{t \in \operatorname{supp}_{2}(x)} 2^{t}$ and $\operatorname{supp}_{2}(0)=\emptyset$.

If $S_{0}=\omega$, let $\psi: \omega \rightarrow \omega$ be the identity. If $S_{0}$ is a countably infinite group which can be mapped into a compact metrizable group by an injective homomorphism, then by [6, Theorem 7.28] we may pick $\psi: \omega \underset{\text { onto }}{\stackrel{1-1}{\longrightarrow}} S$ such that $\psi(0)=0$, the restriction of $\widetilde{\psi}$ to $\mathbb{H}$ is an injective homomorphism, and all idempotents of $\beta S \backslash S$ are in $\widetilde{\psi}[\mathbb{H}]$. In any event the hypotheses of Lemma 2.6 are satisfied. 
Notice that $|S|=\mathfrak{c}$, and so if $\mathcal{T}={ }^{\mathbb{N}} S$, we have $\left|\mathcal{P}_{\mathrm{f}}(\mathcal{T})\right|=\mathfrak{c}$. Enumerate $\mathcal{P}_{\mathrm{f}}(\mathcal{T})$ as $\left\langle F_{\sigma}\right\rangle_{\sigma<\mathfrak{c}}$ and for each $\sigma<\mathfrak{c}$, let $m(\sigma)=\left|F_{\sigma}\right|$. Write

$$
F_{\sigma}=\left\{\left\langle y_{\sigma, i, t}\right\rangle_{t=1}^{\infty}: i \in\{1, \ldots, m(\sigma)\}\right\} .
$$

Let $\left\{E_{n}: n \in \mathbb{N}\right\}$ be a partition of $\mathbb{N}$ into infinite sets and define $\theta: \mathbb{N} \rightarrow \mathbb{N}$ by $n \in E_{\theta(n)}$. Let $D=\{\sigma<\mathfrak{c}: \sigma$ is a limit ordinal $\}$ and choose $\gamma: \mathfrak{c} \stackrel{1-1}{\longrightarrow} D$ such that for all $\sigma<\mathfrak{c}$,

$$
\sup \left(\bigcup_{i=1}^{m(\sigma)} \bigcup_{t=1}^{\infty} \operatorname{supp}\left(y_{\sigma, i, t}\right)\right)<\gamma(\sigma) .
$$

We choose inductively for $\sigma<\mathfrak{c}$ sequences $\left\langle a_{\sigma, n}\right\rangle_{n=1}^{\infty}$ in $S$ and $\left\langle H_{\sigma, n}\right\rangle_{n=1}^{\infty}$ in $\mathcal{P}_{\mathrm{f}}(\mathbb{N})$ as follows. Let $\sigma<\mathfrak{c}$ be given and assume $\left\langle a_{\tau, n}\right\rangle_{n=1}^{\infty}$ and $\left\langle H_{\tau, n}\right\rangle_{n=1}^{\infty}$ have been chosen for all $\tau<\sigma$. Choose $k_{1} \in E_{m(\sigma)}$ and $H_{\sigma, 1} \in \mathcal{P}_{\mathrm{f}}(\mathbb{N})$ such that for each $i \in\{1, \ldots, m(\sigma)\}, 2^{k_{1}+1}$ divides $\psi^{-1}\left(\sum_{t \in H_{\sigma, 1}} \pi_{0}\left(y_{\sigma, i, t}\right)\right)=$ $\psi^{-1}\left(\pi_{0}\left(\sum_{t \in H_{\sigma, 1}} y_{\sigma, i, t}\right)\right)$, which one can do by Lemma 2.6. Choose $k_{2} \in E_{m(\sigma)}$ with $\psi^{-1}\left(\pi_{0}\left(\sum_{t \in H_{\sigma, 1}} y_{\sigma, i, t}\right)\right)<2^{k_{2}}$ for each $i \in\{1, \ldots, m(\sigma)\}$ and $k_{2}>k_{1}$. (The last inequality is redundant unless $\pi_{0}\left(\sum_{t \in H_{\sigma, 1}} y_{\sigma, i, t}\right)=0$ for each $i \in$ $\{1, \ldots, m(\sigma)\}$, which is possible.) Let

$$
a_{\sigma, 1}=\psi\left(2^{k_{1}}+2^{k_{2}}\right) \cdot e(0)+e(\gamma(\sigma)+1) .
$$

Now let $n \in \mathbb{N}$ and assume that $a_{\sigma, n}$ and $H_{\sigma, n}$ have been chosen. Pick $k_{2 n+1} \in E_{m(\sigma)}$ such that $k_{2 n+1}>k_{2 n}$ and pick $H_{\sigma, n+1} \in \mathcal{P}_{\mathrm{f}}(\mathbb{N})$ such that $\min H_{\sigma, n+1}>\max H_{\sigma, n}$ and $2^{k_{2 n+1}+1}$ divides $\psi^{-1}\left(\pi_{0}\left(\sum_{t \in H_{\sigma, n+1}} y_{\sigma, i, t}\right)\right)$ for each $i \in\{1, \ldots, m(\sigma)\}$. Pick $k_{2 n+2} \in E_{m(\sigma)}$ such that $k_{2 n+2}>k_{2 n+1}$ and $\psi^{-1}\left(\pi_{0}\left(\sum_{t \in H_{\sigma, n+1}} y_{\sigma, i, t}\right)\right)<2^{k_{2 n+2}}$ for each $i \in\{1, \ldots, m(\sigma)\}$. Let

$$
a_{\sigma, n+1}=\psi\left(2^{k_{2 n+1}}+2^{k_{2 n+2}}\right) \cdot e(0)+e(\gamma(\sigma)+n+1) .
$$

Now for each $\sigma<\mathfrak{c}$, let $A_{\sigma}=\bigcup_{i=1}^{m(\sigma)} \operatorname{FS}\left(\left\langle x\left(a_{\sigma, n}, H_{\sigma, n},\left\langle y_{\sigma, i, t}\right\rangle_{t=1}^{\infty}\right)\right\rangle_{n=1}^{\infty}\right)$ and $A=\bigcup_{\sigma<\mathfrak{c}} A_{\sigma}$.

Observe that if $\sigma<\mathfrak{c}$ and $x \in A_{\sigma}$, then

(1) $\theta\left(\min \operatorname{supp}_{2} \psi^{-1}\left(\pi_{0}(x)\right)\right)=\theta\left(\max _{\operatorname{supp}_{2}} \psi^{-1}\left(\pi_{0}(x)\right)\right)=m(\sigma)$,

(2) there exist $i \in\{1, \ldots, m(\sigma)\}$ and $G \in \mathcal{P}_{\mathrm{f}}(\mathbb{N})$ such that

$$
x=\sum_{n \in G} x\left(a_{\sigma, n}, H_{\sigma, n},\left\langle y_{\sigma, i, t}\right\rangle_{t=1}^{\infty}\right)
$$

where

(a) $\gamma(\sigma)<\max \operatorname{supp}(x)<\gamma(\sigma)+\omega$,

(b) $\operatorname{supp}(x) \cap(\gamma(\sigma), \gamma(\sigma)+\omega)=\{\gamma(\sigma)+n: n \in G\}$.

We see directly that $A$ satisfies the conclusion of Theorem 2.4. Suppose that $A$ satisfies the conclusion of Theorem 1.4. Let $f_{1}(n)=e(0)$ and $f_{2}(n)=$ 
$2 \cdot e(0)$ for each $n \in \mathbb{N}$. Pick sequences $\left\langle a_{n}\right\rangle_{n=1}^{\infty}$ in $S$ and $\left\langle H_{n}\right\rangle_{n=1}^{\infty}$ in $\mathcal{P}_{\mathrm{f}}(\mathbb{N})$ such that $\max H_{n}<\min H_{n+1}$ for each $n \in \mathbb{N}$ and whenever $K \in \mathcal{P}_{\mathrm{f}}(\mathbb{N})$ and $g: K \rightarrow\{1,2\}$, then

$$
\sum_{n \in K} x\left(a_{n}, H_{n}, f_{g(n)}\right) \in A .
$$

For $r \in \mathbb{N} \backslash\{1\}$ and $l \in\{2, \ldots, r\}$, let

$$
b(l, r)=\sum_{n=2}^{l} x\left(a_{n}, H_{n}, f_{1}\right)+\sum_{n=l+1}^{r+1} x\left(a_{n}, H_{n}, f_{2}\right)
$$

and note that for each $l \in\{2, \ldots, r-1\}, \pi_{0}(b(l, r))>\pi_{0}(b(l+1, r))$.

Now let $B=\left\{1, \ldots, \max \operatorname{supp}_{2} \psi^{-1}\left(\pi_{0}\left(x\left(a_{1}, H_{1}, f_{1}\right)\right)\right)\right\}$. We claim that $\left\{\theta\left(\max _{\operatorname{supp}} \psi^{-1}\left(\pi_{0}(b(l, r))\right)\right): r \in \mathbb{N} \backslash\{1\}\right.$ and $\left.l \in\{2, \ldots, r\}\right\} \subseteq \theta[B]$. To see this, let $r \in \mathbb{N} \backslash\{1\}$ and $l \in\{2, \ldots, r\}$. If

$$
\min \operatorname{supp}_{2} \psi^{-1}\left(\pi_{0}(b(l, r))\right) \leq \max _{\operatorname{supp}_{2}} \psi^{-1}\left(\pi_{0}\left(x\left(a_{1}, H_{1}, f_{1}\right)\right)\right),
$$

then $\min \operatorname{supp}_{2} \psi^{-1}\left(\pi_{0}(b(l, r))\right) \in B$, so

$$
\theta\left(\max _{\operatorname{supp}_{2}} \psi^{-1}\left(\pi_{0}(b(l, r))\right)\right)=\theta\left(\min _{\operatorname{supp}_{2}} \psi^{-1}\left(\pi_{0}(b(l, r))\right)\right) \in \theta[B] .
$$

So assume $\min \operatorname{supp}_{2} \psi^{-1}\left(\pi_{0}(b(l, r))\right)>\max \operatorname{supp}_{2} \psi^{-1}\left(\pi_{0}\left(x\left(a_{1}, H_{1}, f_{1}\right)\right)\right)$ and let $x=x\left(a_{1}, H_{1}, f_{1}\right)+b(l, r)$. Then $x \in A_{\sigma}$ for some $\sigma$, so

$$
\begin{aligned}
\theta\left(\max _{\operatorname{supp}} \psi^{-1}\left(\pi_{0}(b(l, r))\right)\right) & =\theta\left(\max _{\left.\operatorname{supp}_{2} \psi^{-1}\left(\pi_{0}(x)\right)\right)}=\theta\left(\min _{\left.\operatorname{supp}_{2} \psi^{-1}\left(\pi_{0}(x)\right)\right)}=\theta\left(\min \operatorname{supp}_{2} \psi^{-1}\left(\pi_{0}\left(x\left(a_{1}, H_{1}, f_{1}\right)\right)\right)\right) \in \theta[B] .\right.\right.
\end{aligned}
$$

Now let $k=\max \theta[B]$ and let $r=k+2$. For each $l \in\{2, \ldots, r\}$ and any $\iota$ with $0<\iota<\mathfrak{c}, \pi_{\iota}(b(l, r))=\pi_{\iota}\left(\sum_{n=2}^{r+1} a_{n}\right)$, which is independent of $l$. Thus by observation (2) there exist $\sigma<\mathfrak{c}$ and $G \in \mathcal{P}_{\mathrm{f}}(\mathbb{N})$ such that for each $l \in\{2, \ldots, r\}$ there is some $i \in\{1, \ldots, m(\sigma)\}$ such that $b(l, r)=$ $\sum_{n \in G} x\left(a_{\sigma, n}, H_{\sigma, n},\left\langle y_{\sigma, i, t}\right\rangle_{t=1}^{\infty}\right)$. Further, since $b(2, r) \in A_{\sigma}$, we have $m(\sigma)=$ $\theta\left(\max \operatorname{supp}_{2} \psi^{-1}\left(\pi_{0}(b(2, r))\right)\right) \leq k$. But we have seen that

$$
\pi_{0}(b(2, r))>\pi_{0}(b(3, r))>\cdots>\pi_{0}(b(r, r)),
$$

so $|\{b(l, r): l \in\{2, \ldots, r\}\}|=k+1$ while

$$
\left|\left\{\sum_{n \in G} x\left(a_{\sigma, n}, H_{\sigma, n},\left\langle y_{\sigma, i, t}\right\rangle_{t=1}^{\infty}\right): i \in\{1, \ldots, m(\sigma)\}\right\}\right| \leq m(\sigma) \leq k .
$$

This contradiction completes the proof.

2.8. Corollary. There is a subset of $(\mathbb{R},+)$ which satisfies the conclusion of Theorem 2.4 but not the conclusion of Theorem 1.4.

Proof. $(\mathbb{R},+)$ is isomorphic to $\bigoplus_{\iota<\mathfrak{c}} \mathbb{Q}$ and $(\mathbb{Q},+)$ can be mapped into the circle group $\mathbb{T}$ by an injective homomorphism.

We ask the following question in the broadest terms, but we do not know the answer for $S=\mathbb{N}$ or $S=\mathbb{R}$. 
2.9. Question. Do there exist a commutative semigroup $(S,+)$ and a subset $C$ of $S$ establishing that the conclusions of Theorems 1.4, 1.6, and 2.2 are not all equivalent?

We shall see in Section 4 that the noncommutative versions of these theorems are strictly increasing in strength.

3. Rich sets, strongly rich sets, and the new noncommutative Central Sets Theorem. As is customary, we use multiplicative notation for a not necessarily commutative semigroup. The versions of the noncommutative Central Sets Theorem are more complicated because the translates $a_{n}$ or $\alpha(F)$ must be split into several parts. That is the function of the notion $\mathcal{I}_{m}$ which we introduce now.

3.1. Definition. For $m \in \mathbb{N}, \mathcal{I}_{m}=\{(H(1), \ldots, H(m))$ : each $H(j) \in$ $\mathcal{P}_{\mathrm{f}}(\mathbb{N})$ and for any $\left.j \in\{1, \ldots, m-1\}, \max H(j)<\min H(j+1)\right\}$.

The following is the version of the noncommutative Central Sets Theorem given in [6]. In a noncommutative semigroup, by $\prod_{t \in F} x_{t}$ we mean the product taken in increasing order of indices.

3.2. Theorem. Let $(S, \cdot)$ be a semigroup, let $C$ be a central subset of $S$, and for each $l \in \mathbb{N}$, let $\left\langle y_{l, i}\right\rangle_{i=1}^{\infty}$ be a sequence in $S$. There exist sequences $\langle m(n)\rangle_{n=1}^{\infty},\langle a(n)\rangle_{n=1}^{\infty}$, and $\langle H(n)\rangle_{n=1}^{\infty}$ such that

(1) for each $n \in \mathbb{N}, m(n) \in \mathbb{N}, a(n) \in S^{m(n)+1}, H(n) \in \mathcal{I}_{m(n)}$, and $\max H(n)(m(n))<\min H(n+1)(1)$,

(2) for each $f \in \Phi$ and $F \in \mathcal{P}_{\mathrm{f}}(\mathbb{N})$,

$$
\prod_{n \in F}\left(\prod_{j=1}^{m(n)}\left(a(n)(j) \cdot \prod_{t \in H(n)(j)} y_{f(n), t}\right)\right) \cdot a(n)(m(n)+1) \in C .
$$

Proof. [6, Theorem 14.15].

In [5] it was shown that central sets were not the only sets satisfying the conclusion of the Central Sets Theorem (Theorem 1.4) in commutative semigroups. Sets satisfying the conclusion of Theorem 1.4 were called rich and it was shown that any quasi-central set, i.e., a set which is a member of an idempotent in the closure of the smallest ideal, is rich. Further it was shown that in $(\mathbb{N},+)$ there are quasi-central sets which are not central and there are rich sets which are not quasi-central.

In this section, we extend the notion of rich to arbitrary semigroups, and introduce the notion of strongly rich. The new stronger Central Sets Theorem (Corollary 3.10) is the assertion that any central set is strongly rich. We show that there is a closed two-sided ideal $J$ of $\beta S$ such that a set is strongly rich if and only if it is a member of an idempotent in $J$. 
We introduce some special notation. The notation does not reflect all of the variables upon which it depends.

3.3. Definition. Let $(S, \cdot)$ be a semigroup.

(a) $\mathcal{T}=\mathbb{N}_{S}$.

(b) $\mathcal{Y}=\mathbb{N}_{\mathcal{T}}$.

(c) Given $m \in \mathbb{N}, a \in S^{m+1}, H \in \mathcal{I}_{m}$, and $f \in \mathcal{T}$,

$$
x(m, a, H, f)=\left(\prod_{j=1}^{m}\left(a(j) \cdot \prod_{t \in H(j)} f(t)\right)\right) \cdot a(m+1) .
$$

(d) Given $Y=\left\langle\left\langle y_{l, t}\right\rangle_{t=1}^{\infty}\right\rangle_{l=1}^{\infty} \in \mathcal{Y}$ and $A \subseteq S, A$ is a $J_{Y}$-set if and only if for all $n \in \mathbb{N}$ there exist $m \in \mathbb{N}, a \in S^{m+1}$, and $H \in \mathcal{I}_{m}$ such that $\min H(1) \geq n$ and for all $l \in\{1, \ldots, n\}, x\left(m, a, H,\left\langle y_{l, t}\right\rangle_{t=1}^{\infty}\right) \in A$.

(e) $A \subseteq S$ is a $J$-set if and only if for each $F \in \mathcal{P}_{\mathrm{f}}(\mathcal{T})$ and each $n \in \mathbb{N}$, there exist $m \in \mathbb{N}, a \in S^{m+1}$, and $H \in \mathcal{I}_{m}$ such that $\min H(1) \geq n$ and for each $f \in F, x(m, a, H, f) \in A$.

(f) Given $Y \in \mathcal{Y}, J_{Y}=\left\{p \in \beta S\right.$ : for all $A \in p, A$ is a $\left.J_{Y^{-} \text {-set }}\right\}$.

(g) $J=\{p \in \beta S$ : for all $A \in p, A$ is a $J$-set $\}$.

(h) $A \subseteq S$ is rich iff for each $Y=\left\langle\left\langle y_{l, i}\right\rangle_{i=1}^{\infty}\right\rangle_{l=1}^{\infty} \in \mathcal{Y}$, there exist sequences $\langle m(n)\rangle_{n=1}^{\infty},\langle a(n)\rangle_{n=1}^{\infty}$, and $\langle H(n)\rangle_{n=1}^{\infty}$ such that

(1) for each $n \in \mathbb{N}, m(n) \in \mathbb{N}, a(n) \in S^{m(n)+1}, H(n) \in \mathcal{I}_{m(n)}$, and $\max H(n)(m(n))<\min H(n+1)(1)$,

(2) for each $f \in \Phi$ and $F \in \mathcal{P}_{\mathrm{f}}(\mathbb{N})$,

$$
\prod_{n \in F} x\left(m(n), a(n), H(n),\left\langle y_{f(n), t}\right\rangle_{t=1}^{\infty}\right) \in A .
$$

(i) $A \subseteq S$ is strongly rich if and only if there exist $m: \mathcal{P}_{\mathrm{f}}(\mathcal{T}) \rightarrow \mathbb{N}$, $\alpha \in \chi_{F \in \mathcal{P}_{\mathrm{f}}(\mathcal{T})} S^{m(F)+1}$, and $H \in \chi_{F \in \mathcal{P}_{\mathrm{f}}(\mathcal{T})} \mathcal{I}_{m(F)}$ such that

(1) if $F, G \in \mathcal{P}_{\mathrm{f}}(\mathcal{T})$ and $F \subsetneq G$, then

$$
\max H(F)(m(F))<\min H(G)(1),
$$

(2) whenever $n \in \mathbb{N}, G_{1}, \ldots, G_{n} \in \mathcal{P}_{\mathrm{f}}(\mathcal{T}), G_{1} \subsetneq \ldots \subsetneq G_{n}$, and for each $i \in\{1, \ldots, n\},\left\langle y_{i, t}\right\rangle_{t=1}^{\infty} \in G_{i}$, one has

$$
\prod_{i=1}^{n} x\left(m\left(G_{i}\right), \alpha\left(G_{i}\right), H\left(G_{i}\right),\left\langle y_{i, t}\right\rangle_{t=1}^{\infty}\right) \in A .
$$

We omit the routine proof of the following theorem.

3.4. Theorem. Let $S$ be a semigroup and let $A \subseteq S$. Then $A$ is a J-set if and only if for each $Y \in \mathcal{Y}, A$ is a $J_{Y}$-set. In particular, $J=\bigcap_{Y \in \mathcal{Y}} J_{Y}$.

3.5. TheOrem. For each $Y \in \mathcal{Y}, J_{Y}$ is a closed two-sided ideal of $\beta S$. Consequently, $J$ is a closed two-sided ideal of $\beta S$ and so $\operatorname{cl} K(\beta S) \subseteq J$. 
Proof. Let $Y \in \mathcal{Y}$. By Theorem 3.2 any idempotent in $K(\beta S)$ is in $J_{Y}$ and thus $J_{Y} \neq \emptyset$. If $p \in \beta S \backslash J_{Y}$, pick $A \in p$ such that $A$ is not a $J_{Y \text {-set. }}$ Then $\bar{A}$ is a neighborhood of $p$ missing $J_{Y}$. Thus $J_{Y}$ is closed.

Now let $p \in J_{Y}$ and let $q \in \beta S$. To see that $p \cdot q \in J_{Y}$, let $A \in p \cdot q$ and let $n \in \mathbb{N}$. Let $B=\left\{z \in S: z^{-1} A \in q\right\}$. Then $B \in p$, so pick $m \in \mathbb{N}$, $a \in S^{m+1}$, and $H \in \mathcal{I}_{m}$ such that $\min H(1) \geq n$ and for all $l \in\{1, \ldots, n\}$, $x\left(m, a, H,\left\langle y_{l, t}\right\rangle_{t=1}^{\infty}\right) \in B$. Pick $z \in \bigcap_{l=1}^{n} x\left(m, a, H,\left\langle y_{l, t}\right\rangle_{t=1}^{\infty}\right)^{-1} A$. Define $b \in$ $S^{m+1}$ by, for $t \in\{1, \ldots, m+1\}$,

$$
b(t)= \begin{cases}a(t) & \text { if } t \leq m, \\ a(m+1) \cdot z & \text { if } t=m+1 .\end{cases}
$$

Then for all $l \in\{1, \ldots, n\}, x\left(m, b, H,\left\langle y_{l, t}\right\rangle_{t=1}^{\infty}\right) \in A$.

To see that $q \cdot p \in J_{Y}$, let $A \in q \cdot p$ and $n \in \mathbb{N}$. Let $B=\{z \in S$ : $\left.z^{-1} A \in p\right\}$. Then $B \in q$ and is therefore nonempty, so pick $z \in B$. Pick $m \in$ $\mathbb{N}, a \in S^{m+1}$, and $H \in \mathcal{I}_{m}$ such that $\min H(1) \geq n$ and for all $l \in\{1, \ldots, n\}$, $x\left(m, a, H,\left\langle y_{l, t}\right\rangle_{t=1}^{\infty}\right) \in z^{-1} A$. Define $b \in S^{m+1}$ by, for $t \in\{1, \ldots, m+1\}$,

$$
b(t)= \begin{cases}z \cdot a(1) & \text { if } t=1, \\ a(t) & \text { if } t \geq 2 .\end{cases}
$$

Then for all $l \in\{1, \ldots, n\}, x\left(m, b, H,\left\langle y_{l, t}\right\rangle_{t=1}^{\infty}\right) \in A$.

We note now a strong relationship between rich sets and the ideals $J_{Y}$ and between strongly rich sets and the ideal $J$.

3.6. Theorem. Let $(S, \cdot)$ be a semigroup and let $A \subseteq S$. Then $A$ is rich if and only if for every $Y \in \mathcal{Y}$ there is an idempotent $p \in J_{Y} \cap \bar{A}$.

Proof. For $S$ commutative, this is [5, Corollary 2.11]. The adjustments to the proof needed for the general case can be deduced from the proof of Theorem 3.8 below, which we present in full detail.

We shall need the following lemma from [6].

3.7. Lemma. Let $P$ be a set, let $(D, \leq)$ be a directed set, and let $(S, \cdot)$ be a semigroup. Let $\left\langle T_{i}\right\rangle_{i \in D}$ be a decreasing family of nonempty subsets of $S$ such that for each $i \in D$ and each $x \in T_{i}$ there is some $j \in D$ such that $x \cdot T_{j} \subseteq T_{i}$. Let $\mathbf{Q}=\bigcap_{i \in D} \operatorname{cl}_{\beta S} T_{i}$. Then $\mathbf{Q}$ is a compact subsemigroup of $\beta S$. Let $\left\langle E_{i}\right\rangle_{i \in D}$ and $\left\langle I_{i}\right\rangle_{i \in D}$ be decreasing families of nonempty subsets of $\chi_{t \in P} S$ with the following properties:

(a) for each $i \in D, I_{i} \subseteq E_{i} \subseteq \mathrm{X}_{t \in P} T_{i}$,

(b) for each $i \in D$ and $x \in I_{i}$ there exists $j \in D$ such that $x \cdot E_{j} \subseteq I_{i}$,

(c) for each $i \in D$ and $x \in E_{i} \backslash I_{i}$ there exists $j \in D$ such that $x \cdot E_{j} \subseteq E_{i}$ and $x \cdot I_{j} \subseteq I_{i}$.

Let $Y=\chi_{t \in P} \beta S$, let $E=\bigcap_{i \in D} \operatorname{cl}_{Y} E_{i}$, and let $I=\bigcap_{i \in D} \operatorname{cl}_{Y} I_{i}$. Then $E$ is a subsemigroup of $X_{t \in P} \mathbf{Q}$ and $I$ is an ideal of $E$. If , in addition, either 
(d) for each $i \in D, T_{i}=S$ and $\left\{a \in S: \bar{a} \notin E_{i}\right\}$ is not piecewise syndetic, or

(e) for each $i \in D$ and $a \in T_{i}, \bar{a} \in E_{i}$,

then given any $p \in K(\mathbf{Q})$, one has $\bar{p} \in E \cap K\left(\mathbf{X}_{t \in P} \mathbf{Q}\right)=K(E) \subseteq I$.

Proof. [6, Lemma 14.9]

As the referee has observed, only the "sufficiency" portion of the following theorem (which is the part with the easier proof) is needed for the corollaries that follow.

3.8. Theorem. Let $(S, \cdot)$ be a semigroup and let $A \subseteq S$. Then $A$ is strongly rich if and only if there is an idempotent $p \in J \cap \overline{\bar{A}}$.

Proof. Sufficiency. Pick $p=p \cdot p \in J \cap \bar{A}$. Recall from the proof of Theorem 2.2 that $A^{\star}=\left\{x \in A: x^{-1} A \in p\right\}$ and if $x \in A^{\star}$, then $x^{-1} A^{\star} \in p$. We define $m(F), \alpha(F)$, and $H(F)$ for $F \in \mathcal{P}_{\mathrm{f}}(\mathcal{T})$ by induction on $|F|$ so that

(1) if $\emptyset \neq G \subsetneq F$, then $\max H(G)(m(G))<\min H(F)(1)$,

(2) whenever $n \in \mathbb{N}, \emptyset \neq G_{1} \subsetneq \cdots \subsetneq G_{n}=F$, and $\tau \in X_{i=1}^{n} G_{i}$, then

$$
\prod_{i=1}^{n} x\left(m\left(G_{i}\right), \alpha\left(G_{i}\right), H\left(G_{i}\right), \tau(i)\right) \in A^{\star} .
$$

Assume first that $F=\{f\}$. Then $A^{\star}$ is a $J$-set, so pick $m(F) \in \mathbb{N}, \alpha(F) \in$ $S^{m(F)+1}$, and $H(F) \in \mathcal{I}_{m(F)}$ such that $x(m(F), \alpha(F), H(F), f) \in A^{\star}$.

Now assume that $|F|>1$ and $m(G), \alpha(G)$, and $H(G)$ have been defined for all proper subsets $G$ of $F$. For $\emptyset \neq G \subsetneq F$, let $l(G)=\max H(G)(m(G))$ and $k=\max \{l(G): \emptyset \neq G \subsetneq F\}+1$. Let

$$
\begin{aligned}
M=\left\{\prod _ { i = 1 } ^ { n } x \left(m\left(G_{i}\right), \alpha\left(G_{i}\right),\right.\right. & \left.H\left(G_{i}\right), \tau(i)\right): \\
& \left.\emptyset \neq G_{1} \subsetneq \cdots \subsetneq G_{n} \subsetneq F \text { and } \tau \in \underset{i=1}{X} G_{i}\right\} .
\end{aligned}
$$

Then $M$ is a finite subset of $A^{\star}$ so $B=A^{\star} \cap \bigcap_{b \in M} b^{-1} A^{\star} \in p$ and so $B$ is a $J$-set. Pick $m(F) \in \mathbb{N}, \alpha(F) \in S^{m(F)+1}$, and $H(F) \in \mathcal{I}_{m(F)}$ such that $\min H(F)(1) \geq k$ and for each $f \in F, x(m(F), \alpha(F), H(F), f) \in B$.

Hypothesis (1) is satisfied directly. To verify (2), let $n \in \mathbb{N}$, let $\emptyset \neq G_{1} \subsetneq$ $\cdots \subsetneq G_{n}=F$, and let $\tau \in X_{i=1}^{n} G_{i}$. If $n=1$, then

$$
x\left(m\left(G_{1}\right), \alpha\left(G_{1}\right), H\left(G_{1}\right), \tau(i)\right) \in B \subseteq A^{\star},
$$

so assume that $n>1$. Let $b=\prod_{i=1}^{n-1} x\left(m\left(G_{i}\right), \alpha\left(G_{i}\right), H\left(G_{i}\right), \tau(i)\right)$. Then $b \in$ $M$ so $x\left(m\left(G_{n}\right), \alpha\left(G_{n}\right), H\left(G_{n}\right), \tau(i)\right) \in B \subseteq b^{-1} A^{\star}$ so $\prod_{i=1}^{n} x\left(m\left(G_{i}\right), \alpha\left(G_{i}\right)\right.$, $\left.H\left(G_{i}\right), \tau(i)\right) \in A^{\star}$ as required. 
Necessity. Pick

$$
m: \mathcal{P}_{\mathrm{f}}(\mathcal{T}) \rightarrow \mathbb{N}, \quad \alpha \in \underset{F \in \mathcal{P}_{\mathrm{f}}(\mathcal{T})}{\chi} S^{m(F)+1}, \quad \text { and } \quad H \in \underset{F \in \mathcal{P}_{\mathrm{f}}(\mathcal{T})}{\chi} \mathcal{I}_{m(F)}
$$

as guaranteed by the fact that $A$ is strongly rich. For $F \in \mathcal{P}_{\mathrm{f}}(\mathcal{T})$ define

$$
\begin{aligned}
T_{F}=\left\{\prod_{i=1}^{n} x\left(m\left(F_{i}\right), \alpha\left(F_{i}\right), H\left(F_{i}\right), \tau(i)\right): n \in \mathbb{N},\right. \\
\left.\quad \text { each } F_{i} \in \mathcal{P}_{\mathrm{f}}(\mathcal{T}), F \varsubsetneqq F_{1} \varsubsetneqq \cdots \varsubsetneqq F_{n}, \text { and } \tau \in \underset{i=1}{\text { X }} F_{i}\right\} .
\end{aligned}
$$

Note that if $F, G \in \mathcal{P}_{\mathrm{f}}(\mathcal{T})$, then $T_{F \cup G} \subseteq T_{F} \cap T_{G}$, so $\mathbf{Q}=\bigcap_{F \in \mathcal{P}_{\mathrm{f}}(\mathcal{T})} \bar{T}_{F}$ $\neq \emptyset$. We claim that $Q$ is a subsemigroup of $\beta S$. For this it suffices by $[6$, Theorem 4.20] to show that for all $F \in \mathcal{P}_{\mathrm{f}}(\mathcal{T})$ and all $u \in T_{F}$, there is some $G \in \mathcal{P}_{\mathrm{f}}(\mathcal{T})$ such that $u \cdot T_{G} \subseteq T_{F}$. So let $F \in \mathcal{P}_{\mathrm{f}}(\mathcal{T})$ and $u \in T_{F}$ be given. Pick strictly increasing $\left\langle F_{i}\right\rangle_{i=1}^{n}$ in $\mathcal{P}_{\mathrm{f}}(\mathcal{T})$ such that $F \subsetneq F_{1}$ and $u=\prod_{i=1}^{n} x\left(m\left(F_{i}\right), \alpha\left(F_{i}\right), H\left(F_{i}\right), \tau(i)\right)$. Then $u \cdot T_{F_{n}} \subseteq T_{F}$.

Now we claim that $K(\mathbf{Q}) \subseteq \bar{A} \cap J$, so that any idempotent in $K(\mathbf{Q})$ establishes the theorem. We see that each $T_{F} \subseteq \bar{A}$ so $\mathbf{Q} \subseteq \bar{A}$. Let $p \in K(\mathbf{Q})$. We need to show that $p \in J$, so let $B \in p$. We shall show that $B$ is a $J$-set. So let $F \in \mathcal{P}_{\mathrm{f}}(\mathcal{T})$ and $k \in \mathbb{N}$ be given. We shall produce $v \in \mathbb{N}, c \in S^{v+1}$, and $M \in \mathcal{I}_{v}$ such that $\min M(1) \geq k$ and for each $f \in F, x(v, c, M, f) \in B$. Note that we can assume that $|F| \geq k$ so if $G \in \mathcal{P}_{\mathrm{f}}(\mathcal{T})$ and $F \subseteq G$, then $\min H(G)(1) \geq k$.

We shall apply Lemma 3.7 with $P=F$ and $D=\left\{G \in \mathcal{P}_{\mathrm{f}}(\mathcal{T}): F \subseteq G\right\}$. Note that $\mathbf{Q}=\bigcap_{G \in D} \bar{T}_{G}$ as in Lemma 3.7. For $G \in D$ we shall define a subset $I_{G}$ of $\chi_{f \in F} S$ as follows. Let $w \in \chi_{f \in F} S$. Then $w \in I_{G}$ if and only if there is some $n \in \mathbb{N} \backslash\{1\}$ such that

(1) there exist disjoint nonempty sets $C_{1}$ and $C_{2}$ such that $\{1, \ldots, n\}=$ $C_{1} \cup C_{2}$,

(2) there exist strictly increasing $\left\langle G_{i}\right\rangle_{i=1}^{n}$ in $\mathcal{P}_{\mathrm{f}}(\mathcal{T})$ with $G \subsetneq G_{1}$,

(3) there exists $\tau \in X_{i \in C_{1}} G_{i}$,

such that for each $f \in F$, if $\gamma_{f} \in X_{i=1}^{n} G_{i}$ is defined by

$$
\gamma_{f}(i)= \begin{cases}\tau(i) & \text { if } i \in C_{1}, \\ f & \text { if } i \in C_{2},\end{cases}
$$

then $w(f)=\prod_{i=1}^{n} x\left(m\left(G_{i}\right), \alpha\left(G_{i}\right), H\left(G_{i}\right), \gamma_{f}(i)\right)$.

For $G \in D$, let $E_{G}=I_{G} \cup\left\{\bar{b}: b \in T_{G}\right\}$.

We claim that $\left\langle E_{G}\right\rangle_{G \in D}$ and $\left\langle I_{G}\right\rangle_{G \in D}$ satisfy statements (a), (b), (c), and (e) of Lemma 3.7. Statements (a) and (e) hold trivially.

To verify (b), let $G \in D$ and $w \in I_{G}$. Pick $n, C_{1}, C_{2},\left\langle G_{i}\right\rangle_{i=1}^{n}$, and $\tau$ as guaranteed by the fact that $w \in I_{G}$. We claim that $w \cdot E_{G_{n}} \subseteq I_{G}$. So let $z \in E_{G_{n}}$. 
Assume first that $z=\bar{b}$ for some $b \in T_{G_{n}}$. Pick $n^{\prime} \in \mathbb{N}$, strictly increasing $\left\langle F_{i}\right\rangle_{i=1}^{n^{\prime}}$ in $\mathcal{P}_{\mathrm{f}}(\mathcal{T})$ with $G_{n} \subsetneq F_{1}$, and $\tau^{\prime} \in \mathrm{X}_{i=1}^{n^{\prime}} F_{i}$ such that

$$
b=\prod_{i=1}^{n^{\prime}} x\left(m\left(F_{i}\right), \alpha\left(F_{i}\right), H\left(F_{i}\right), \tau^{\prime}(i)\right) .
$$

Let $C_{1}^{\prime \prime}=C_{1} \cup\left\{n+1, \ldots, n+n^{\prime}\right\}$, and for $i \in\left\{1, \ldots, n+n^{\prime}\right\}$, let

$$
L_{i}= \begin{cases}G_{i} & \text { if } i \leq n, \\ F_{i-n} & \text { if } i>n .\end{cases}
$$

Define $\tau^{\prime \prime} \in X_{i \in C_{1}^{\prime \prime}} L_{i}$ by setting, for $i \in C_{1}^{\prime \prime}$,

$$
\tau^{\prime \prime}(i)= \begin{cases}\tau(i) & \text { if } i \leq n, \\ \tau^{\prime}(i-n) & \text { if } i>n .\end{cases}
$$

Then $n+n^{\prime}, C_{1}^{\prime \prime}, C_{2},\left\langle L_{i}\right\rangle_{i=1}^{n+n^{\prime}}$, and $\tau^{\prime \prime}$ establish that $w \cdot z \in I_{G}$.

Now assume that $z \in I_{G_{n}}$. Pick $n^{\prime}, C_{1}^{\prime}, C_{2}^{\prime},\left\langle F_{i}\right\rangle_{i=1}^{n^{\prime}}$, and $\tau^{\prime}$ as guaranteed by the fact that $z \in I_{G_{n}}$. Let $C_{1}^{\prime \prime}=C_{1} \cup\left\{n+i: i \in C_{1}^{\prime}\right\}$, let $C_{2}^{\prime \prime}=C_{2} \cup\{n+i$ : $\left.i \in C_{2}^{\prime}\right\}$, and for $i \in\left\{1, \ldots, n+n^{\prime}\right\}$ let

$$
L_{i}= \begin{cases}G_{i} & \text { if } i \leq n \\ F_{i-n} & \text { if } i>n\end{cases}
$$

Define $\tau^{\prime \prime} \in X_{i \in C_{1}^{\prime \prime}} L_{i}$ by setting, for $i \in C_{1}^{\prime \prime}$,

$$
\tau^{\prime \prime}(i)= \begin{cases}\tau(i) & \text { if } i \leq n, \\ \tau^{\prime}(i-n) & \text { if } i>n .\end{cases}
$$

Then $n+n^{\prime}, C_{1}^{\prime \prime}, C_{2}^{\prime \prime},\left\langle L_{i}\right\rangle_{i=1}^{n+n^{\prime}}$, and $\tau^{\prime \prime}$ establish that $w \cdot z \in I_{G}$.

To verify (c) let $G \in D$ and $w \in E_{G} \backslash I_{G}$. Pick $b \in T_{G}$ such that $w=\bar{b}$. Pick $n \in \mathbb{N}$, strictly increasing $\left\langle G_{i}\right\rangle_{i=1}^{n}$ in $\mathcal{P}_{\mathrm{f}}(\mathcal{T})$ with $G \subsetneq G_{1}$, and $\tau \in X_{i=1}^{n} G_{i}$ such that $b=\prod_{i=1}^{n} x\left(m\left(G_{i}\right), \alpha\left(G_{i}\right), H\left(G_{i}\right), \tau(i)\right)$. Then as above one has $w \cdot E_{G_{n}} \subseteq E_{G}$ and $w \cdot I_{G_{n}} \subseteq I_{G}$.

It follows from Lemma 3.7 that $\bar{p} \in I=\bigcap_{G \in D} \bar{I}_{G}$. Now $\chi_{f \in F} \bar{B}$ is a neighborhood of $\bar{p}$ so pick $w \in I_{F} \cap \chi_{f \in F} \bar{B}$. Pick $n, C_{1}, C_{2},\left\langle G_{i}\right\rangle_{i=1}^{n}$, and $\tau \in \mathbf{X}_{i \in C_{1}} G_{i}$ as guaranteed by the fact that $w \in I_{F}$. Let $r=\left|C_{2}\right|$ and let $h_{1}, \ldots, h_{r}$ be the elements of $C_{2}$ listed in increasing order. Let $v=$ $\sum_{i=1}^{r} m\left(G_{h_{i}}\right)$. If $h_{1}=1$, let $c(1)=\alpha\left(G_{1}\right)(1)$. If $h_{1}>1$, let

$$
c(1)=\prod_{i=1}^{h_{1}-1}\left(x\left(m\left(G_{i}\right), \alpha\left(G_{i}\right), H\left(G_{i}\right), \tau(i)\right)\right) \cdot \alpha\left(G_{h_{1}}\right)(1) .
$$

For $1<j \leq m\left(G_{h_{1}}\right)$ let $c(j)=\alpha\left(G_{h_{1}}\right)(j)$ and $M(j)=H\left(G_{h_{1}}\right)(j)$.

Now let $s \in\{1, \ldots, r-1\}$ and $u=\sum_{i=1}^{s} m\left(G_{h_{i}}\right)$. If $h_{s+1}=h_{s}+1$ let

$$
c(u+1)=\alpha\left(G_{h_{s}}\right)\left(m\left(G_{h_{s}}\right)+1\right) \cdot \alpha\left(G_{h_{s+1}}\right)(1) .
$$


If $h_{s+1}>h_{s}+1$, let

$c(u+1)$

$=\alpha\left(G_{h_{s}}\right)\left(m\left(G_{h_{s}}\right)+1\right) \cdot\left(\prod_{i=h_{s}+1}^{h_{s+1}-1} x\left(m\left(G_{i}\right), \alpha\left(G_{i}\right), H\left(G_{i}\right), \tau(i)\right)\right) \cdot \alpha\left(G_{h_{s+1}}\right)(1)$.

And for $u<j \leq \sum_{i=1}^{s+1} m\left(G_{h_{i}}\right)$, let $M(j)=H\left(G_{h_{s+1}}\right)(j-u)$.

If $h_{r}=n$, let $c(v+1)=\alpha\left(G_{n}\right)\left(m\left(G_{n}\right)+1\right)$. If $h_{r}<n$, let

$$
c(v+1)=\alpha\left(G_{h_{r}}\right)\left(m\left(G_{h_{r}}\right)+1\right) \cdot \prod_{i=h_{r}+1}^{n}\left(x\left(m\left(G_{i}\right), \alpha\left(G_{i}\right), H\left(G_{i}\right), \tau(i)\right)\right) .
$$

Then $c \in S^{v+1}, M \in \mathcal{I}_{v}$ such that $\min M(1) \geq k$, and for each $f \in F$, $x(v, c, M, f) \in B$ as required.

3.9. Corollary. Let $(S, \cdot)$ be a semigroup. Every quasi-central subset of $S$ is strongly rich.

Proof. Apply Theorems 3.5 and 3.8.

We isolate the following corollary with a full statement of the conclusion because it is the new Central Sets Theorem.

3.10. Corollary. Let $(S, \cdot)$ be a semigroup and let $C$ be a central subset of $S$. There exist $m: \mathcal{P}_{\mathrm{f}}(\mathcal{T}) \rightarrow \mathbb{N}, \alpha \in \chi_{F \in \mathcal{P}_{\mathrm{f}}(\mathcal{T})} S^{m(F)+1}$, and $H \in \chi_{F \in \mathcal{P}_{\mathrm{f}}(\mathcal{T})} \mathcal{I}_{m(F)}$ such that

(1) if $F, G \in \mathcal{P}_{\mathrm{f}}(\mathcal{T})$ and $F \subsetneq G$, then $\max H(F)(m(F))<\min H(G)(1)$,

(2) whenever $n \in \mathbb{N}, G_{1}, \ldots, G_{n} \in \mathcal{P}_{\mathrm{f}}(\mathcal{T}), G_{1} \subsetneq \cdots \subsetneq G_{n}$, and for each $i \in\{1, \ldots, n\},\left\langle y_{i, t}\right\rangle_{t=1}^{\infty} \in G_{i}$, one has

$$
\prod_{i=1}^{n} x\left(m\left(G_{i}\right), \alpha\left(G_{i}\right), H\left(G_{i}\right),\left\langle y_{i, t}\right\rangle_{t=1}^{\infty}\right) \in C .
$$

Proof. A central set is quasi-central.

Theorem 4.4 below gives an example of a rich set in the free semigroup on $\omega_{1}$ generators which is not strongly rich.

By Theorem 3.8, the example of [5, Theorem 5.5] of a subset of $\mathbb{N}$ which is rich and not quasi-central is in fact strongly rich. By Theorems 3.6 and 3.8 the example given in Theorem 4.4 of a subset of the free semigroup on $\omega_{1}$ generators is a member of an idempotent in $J_{Y}$ for each $Y \in \mathcal{Y}$ but is not a member of any idempotent in $J$.

4. Strength of the versions of the Central Sets Theorem in noncommutative semigroups. Each of Theorems 1.1 and 1.4 has natural noncommutative versions which we now state. They are, of course, each corollaries of Theorem 3.2. 
4.1. Theorem. Let $S$ be a semigroup, let $Z \in \mathcal{P}_{\mathrm{f}}(\mathcal{T})$, and let $C$ be a central subset of $S$. There exist sequences $\langle m(n)\rangle_{n=1}^{\infty},\langle a(n)\rangle_{n=1}^{\infty}$, and $\langle H(n)\rangle_{n=1}^{\infty}$ such that

(1) for each $n \in \mathbb{N}, m(n) \in \mathbb{N}, a(n) \in S^{m(n)+1}, H(n) \in \mathcal{I}_{m(n)}$, and $\max H(n)(m(n))<\min H(n+1)(1)$,

(2) for each $f \in Z$ and $F \in \mathcal{P}_{\mathrm{f}}(\mathbb{N}), \prod_{n \in F} x(m(n), a(n), H(n), f) \in A$.

4.2. Theorem. Let $S$ be a semigroup, let $Z \in \mathcal{P}_{\mathrm{f}}(\mathcal{T})$, and let $C$ be a central subset of $S$. There exist sequences $\langle m(n)\rangle_{n=1}^{\infty},\langle a(n)\rangle_{n=1}^{\infty}$, and $\langle H(n)\rangle_{n=1}^{\infty}$ such that

(1) for each $n \in \mathbb{N}, m(n) \in \mathbb{N}, a(n) \in S^{m(n)+1}, H(n) \in \mathcal{I}_{m(n)}$, and $\max H(n)(m(n))<\min H(n+1)(1)$,

(2) for each $F \in \mathcal{P}_{\mathrm{f}}(\mathbb{N})$ and $f: F \rightarrow Z$,

$$
\prod_{n \in F} x(m(n), a(n), H(n), f(n)) \in A .
$$

We now show that Theorems 4.1, 4.2, 3.2, and Corollary 3.10 are strictly increasing in strength. For the following, recall that any ordinal is the set of its predecessors. In particular, the cardinal $\omega_{1}$ is the set of countable ordinals.

4.3. THEOREM. Let $S$ be the free semigroup on the alphabet $\mathfrak{c}$. There exist subsets $A$ and $B$ of $S$ such that $A$ satisfies the conclusion of Theorem 4.1 but not that of Theorem 4.2, and B satisfies the conclusion of Theorem 4.2 but not that of Theorem 3.2 .

Proof. Enumerate $\mathcal{P}_{\mathrm{f}}(\mathcal{T})$ as $\left\langle Z_{\sigma}\right\rangle_{\sigma<\mathfrak{c}}$. Choose an injective mapping $\sigma \mapsto$ $z_{\sigma}$ from $\mathfrak{c}$ to $\mathfrak{c} \backslash \omega$ such that if $f \in Z_{\sigma}, n \in \mathbb{N}$, and $\delta$ occurs in $f(n)$, then $\delta<z_{\sigma}$.

Let $A_{\sigma}=\left\{\prod_{n \in F} z_{\sigma}^{2^{n}-1} f(n) z_{\sigma}: F \in \mathcal{P}_{\mathrm{f}}(\mathbb{N})\right.$ and $\left.f \in Z_{\sigma}\right\}$ and $A=$ $\bigcup_{\sigma<\mathfrak{c}} A_{\sigma}$. To see that $A$ satisfies the conclusion of Theorem 4.1 , let $Z$ in $\mathcal{P}_{\mathrm{f}}(\mathcal{T})$ be given and pick $\sigma<\mathfrak{c}$ such that $Z=Z_{\sigma}$. For each $n \in \mathbb{N}$, let $m(n)=1, a(n)=\left(z_{\sigma}^{2^{n}-1}, z_{\sigma}\right)$, and $H(n)=(\{n\})$. Then for $n \in \mathbb{N}$ and $f \in Z, x(m(n), a(n), H(n), f)=z_{\sigma}^{2^{n}-1} f(n) z_{\sigma}$, so for each $F \in \mathcal{P}_{\mathrm{f}}(\mathbb{N})$, $\prod_{n \in F} x(m(n), a(n), H(n), f) \in A$.

Now suppose that $A$ satisfies the conclusion of Theorem 4.2. Let $g_{1}(n)$ $=1$ and $g_{2}(n)=2$ for all $n \in \mathbb{N}$ and let $Z=\left\{g_{1}, g_{2}\right\}$. Pick sequences $\langle m(n)\rangle_{n=1}^{\infty},\langle a(n)\rangle_{n=1}^{\infty}$, and $\langle H(n)\rangle_{n=1}^{\infty}$ as guaranteed for $Z$. Pick $\sigma<\mathfrak{c}$ such that $x\left(m(1), a(1), H(1), g_{1}\right) \in A_{\sigma}$. Pick $r \in \mathbb{N}$ such that $2^{r-1}>\left|Z_{\sigma}\right|$. Words in $A_{\sigma}$ begin and end with $z_{\sigma}$. Therefore, given $f:\{1, \ldots, r\} \rightarrow$ $\left\{g_{1}, g_{2}\right\}$ with $f(1)=g_{1}$, there exist $F \in \mathcal{P}_{\mathrm{f}}(\mathbb{N})$ and $h \in Z_{\sigma}$ such that $\prod_{i=1}^{r} x(m(i), a(i), H(i), f(i))=\prod_{n \in F} z_{\sigma}^{2^{n}-1} h(n) z_{\sigma}$. Let $d$ be the number of occurrences of $z_{\sigma}$ in $\prod_{i=1}^{r} \prod_{j=1}^{m(i)+1} a(i)(j)$. Then $d=\sum_{n \in F} 2^{n}$ so $F$ 
does not depend on $f$. But there are $2^{r-1}$ distinct products of the form $\prod_{i=1}^{r} x(m(i), a(i), H(i), f(i))$ where $f:\{1, \ldots, r\} \rightarrow\left\{g_{1}, g_{2}\right\}$ and $f(1)=g_{1}$ while there are only at most $\left|Z_{\sigma}\right|$ distinct products $\prod_{n \in F} z_{\sigma}^{2^{n}-1} h(n) z_{\sigma}$ for $h \in Z_{\sigma}$, a contradiction.

Now let $B_{\sigma}=\left\{\prod_{n \in F} z_{\sigma}^{2^{n}-1} f(n)(n) z_{\sigma}: F \in \mathcal{P}_{\mathrm{f}}(\mathbb{N})\right.$ and $\left.f: F \rightarrow Z_{\sigma}\right\}$ and $B=\bigcup_{\sigma<\mathrm{c}} B_{\sigma}$. To see that $B$ satisfies the conclusion of Theorem 4.2, let $Z \in \mathcal{P}_{\mathrm{f}}(\mathcal{T})$ be given and pick $\sigma<\mathfrak{c}$ such that $Z=Z_{\sigma}$. For each $n \in \mathbb{N}$, let $m(n)=1, a(n)=\left(z_{\sigma}^{2^{n}-1}, z_{\sigma}\right)$, and $H(n)=(\{n\})$. Then for $n \in \mathbb{N}$ and $f: F \rightarrow Z, x(m(n), a(n), H(n), f(n))=z_{\sigma}^{2^{n}-1} f(n)(n) z_{\sigma}$, so for each $F \in \mathcal{P}_{\mathrm{f}}(\mathbb{N})$,

$$
\prod_{n \in F} x(m(n), a(n), H(n), f(n)) \in B .
$$

To see that $B$ does not satisfy the conclusion of Theorem 3.2, for each $l, n \in \mathbb{N}$ let $g_{l}(n)=l$. Suppose we have sequences $\langle m(n)\rangle_{n=1}^{\infty},\langle a(n)\rangle_{n=1}^{\infty}$, and $\langle H(n)\rangle_{n=1}^{\infty}$ such that

(1) for each $n \in \mathbb{N}, m(n) \in \mathbb{N}, a(n) \in S^{m(n)+1}, H(n) \in \mathcal{I}_{m(n)}$, and $\max H(n)(m(n))<\min H(n+1)(1)$,

(2) for each $f \in \Phi$ and $F \in \mathcal{P}_{\mathrm{f}}(\mathbb{N}), \prod_{n \in F} x\left(m(n), a(n), H(n), g_{f(n)}\right) \in B$.

Pick $\sigma<\mathfrak{c}$ such that $b=x\left(m(1), a(1), H(1), g_{1}\right) \in B_{\sigma}$. Pick $r \in \mathbb{N}$ such that $r>\left|Z_{\sigma}\right|$. For $i \in\{1, \ldots, r\}$ let $c_{i}=x\left(m(r), a(r), H(r), g_{i}\right)$. Then for each $i \in\{1, \ldots, r\}, c_{i} \in B$ and $b c_{i} \in B$. Since $b$ begins with $z_{\sigma}$ and each element of $B$ begins and ends with the same letter, $c_{i}$ ends with $z_{\sigma}$ and therefore $c_{i} \in B_{\sigma}$. Assume that $H(r)=\left(L_{1}, \ldots, L_{m(r)}\right)$ and for $j \in$ $\{1, \ldots, m(r)\}$, let $l_{j}=\left|L_{j}\right|$. Then for each $i \in\{1, \ldots, r\}$,

$$
c_{i}=a(r)(1) i^{l_{1}} a(r)(2) i^{l_{2}} \ldots i^{l_{m(r)}} a(r)(m(r)+1) .
$$

Let $d$ be the number of occurrences of $z_{\sigma}$ in $\prod_{j=1}^{m(r)+1} a(r)(j)$. If $d=\sum_{n \in F} 2^{n}$, then for each $i \in\{1, \ldots, r\}$, we have $c_{i}=\prod_{n \in F} z_{\sigma}^{2^{n}-1} h_{i}(n)(n) z_{\sigma}$ for some $h_{i}: F \rightarrow Z_{\sigma}$.

We see that $z_{\sigma}$ occurs in $a(r)(1)$ and in $a(r)(m(r)+1)$. Let $j$ be the least member of $\{2, \ldots, m(r)+1\}$ such that $z_{\sigma}$ occurs in $a(r)(j)$. Then $a(r)(1)=u z_{\sigma}^{2^{n}-1} v$ and $a(r)(j)=w z_{\sigma} y$ where $n \in F, v$ and $w$ are possibly empty words over the letters less than $z_{\sigma}, u$ and $y$ are possibly empty words over the letters less than or equal to $z_{\sigma}$, and $u$ is either empty or ends in a single occurrence of $z_{\sigma}$. (Recall that if $g \in Z_{\sigma}, n \in \mathbb{N}$, and $\delta$ occurs in $g(n)$, then $\delta<z_{\sigma}$.)

Thus for each $i \in\{1, \ldots, r\}$,

$$
z_{\sigma}^{2^{n}-1} h_{i}(n)(n) z_{\sigma}=z_{\sigma}^{2^{n}-1} v i^{l_{1}} a(r)(2) \cdots a(r)(j-1) i^{l_{j-1}} w z_{\sigma} .
$$

Therefore there are $r$ distinct values for $h_{i}(n)(n)$, while each $h_{i}(n) \in Z_{\sigma}$, a contradiction. 
4.4. Theorem. Let $S$ be the free semigroup on the alphabet $\omega_{1}$. There is a subset $C$ of $S$ such that satisfies the conclusion of Theorem 3.2, but not that of Corollary 3.10.

Proof. For each $\lambda<\omega_{1}$, let $S_{\lambda}$ denote the free semigroup on $\left\{\iota<\omega_{1}\right.$ : $\iota \leq \lambda\}$, regarded as a subsemigroup of $S$. Let

$$
C=\bigcup_{\lambda<\omega_{1}} \lambda S_{\lambda}
$$

So $C$ is the set of words $s$ in $S$ whose first letter is greater than or equal to any other letter in $s$. We observe that, for each $\lambda<\omega_{1}, \lambda S_{\lambda}$ is central in $S_{\lambda}$, because it is a right ideal of $S_{\lambda}$. If $Y=\left\langle\left\langle y_{i, t}\right\rangle_{t=1}^{\infty}\right\rangle_{i=1}^{\infty} \in \mathcal{Y}$, we can choose $\lambda<\omega_{1}$ such that $\left\{y_{i, t}: i, t \in \mathbb{N}\right\} \subseteq S_{\lambda}$. It follows from Theorem 3.2 applied to the semigroup $S_{\lambda}$ that $C$ satisfies the conclusion of Theorem 3.2.

We claim that $C$ does not satisfy the conclusion of Corollary 3.10. To see this, suppose that, on the contrary, there exist functions $m, \alpha$ and $H$ satisfying that conclusion. Choose $F=\{f\} \in \mathcal{P}_{\mathrm{f}}(\mathcal{T})$. Then choose $\lambda<\omega_{1}$ such that $x(m(F), \alpha(F), H(F), f) \in \lambda S_{\lambda}$. Choose $\mu$ satisfying $\lambda<\mu<\omega_{1}$. Put $g=\langle\mu, \mu, \ldots\rangle$ and $G=\{f, g\} \in \mathcal{P}_{\mathrm{f}}(\mathcal{T})$.

We are assuming that

$$
s=x(m(F), \alpha(F), H(F), f) x(m(G), \alpha(G), H(G), g) \in \nu S_{\nu}
$$

for some $\nu<\omega_{1}$. This implies that the first letter of $s$ is $\nu$ and hence that $\nu=\lambda$, because the first letter of $s$ is equal to the first letter of $x(m(F), \alpha(F), H(F), f)$. However, $\mu$ occurs in $s$ and hence $\mu \leq \lambda$, a contradiction.

Notice that none of our examples involve a countable semigroup $S$.

4.5. Question. Do there exist a countable semigroup $S$ and a subset $C$ of $S$ satisfying the conclusion of one of Theorems 4.1, 4.2, or 3.2, but not of one or all of the stronger statements?

\section{References}

[1] V. Bergelson and N. Hindman, Nonmetrizable topological dynamics and Ramsey theory, Trans. Amer. Math. Soc. 320 (1990), 293-320.

[2] -, - Ramsey theory in non-commutative semigroups, ibid. 330 (1992), 433-446.

[3] H. Furstenberg, Recurrence in Ergodic Theory and Combinatorial Number Theory, Princeton Univ. Press, Princeton, 1981.

[4] H. Furstenberg and Y. Katznelson, Idempotents in compact semigroups and Ramsey theory, Israel J. Math. 68 (1989), 257-270.

[5] N. Hindman, A. Maleki, and D. Strauss, Central sets and their combinatorial characterization, J. Combin. Theory Ser. A 74 (1996), 188-208.

[6] N. Hindman and D. Strauss, Algebra in the Stone-Čech Compactification: Theory and Applications, de Gruyter, Berlin, 1998. 
[7] R. Rado, Studien zur Kombinatorik, Math. Z. 36 (1933), 242-280.

[8] H. Shi and H. Yang, Nonmetrizable topological dynamical characterization of central sets, Fund. Math. 150 (1996), 1-9.

Department of Mathematics

Krishnagar Women's College

Krishnagar, Nadia-741101

West Bengal, India

E-mail: dibyendude@gmail.com

Department of Pure Mathematics

University of Leeds

Leeds LS2 9J2, UK

E-mail: d.strauss@hull.ac.uk
Department of Mathematics

Howard University

Washington, DC 20059, U.S.A.

E-mail: nhindman@aol.com

Received 2 October 2006;

in revised form 9 August 2007 